\title{
Copper/nickel-decorated olive pit biochar: One pot solid state synthesis for environmental remediation
}

\author{
Ahmed M. Khalil ${ }^{1,2} *$, Laurent Michely ${ }^{2}$, Rémy Pires ${ }^{2}$, Stéphane Bastide $^{2 *}$, \\ Khouloud Jlassi $^{3}$, Souad Ammar ${ }^{4}$, Mohamed Jaziri ${ }^{5}$, Mohamed M. Chehimi ${ }^{2,4 *}$ \\ ${ }^{1}$ Photochemistry Department, National Research Centre, Dokki, 12622 Giza, Egypt \\ ${ }^{2}$ Université Paris Est, CNRS, ICMPE (UMR 7182), 94320 Thiais, France \\ ${ }^{3}$ Center for Advanced Materials, Qatar University, P.O. Box 2713, Doha, Qatar \\ ${ }^{4}$ Université de Paris, CNRS, ITODYS (UMR 7086), 75013 Paris, France \\ ${ }^{5}$ Laboratoire Electrochimie Et Environnement, Ecole Nationale d'Ingénieurs de Sfax, \\ Université de Sfax, 3038 Sfax, Tunisia
}

\section{Corresponding authors:}

Ahmed M. Khalil (akhali175@yahoo.com), https://orcid.org/0000-0002-4252-8175

Stéphane Bastide (bastide@icmpe.cnrs.fr), https://orcid.org/0000-0002-1938-1304

Mohamed M. Chehimi (mmchehimi@ yahoo.fr); https://orcid.org/0000-0002-6098-983X

\begin{abstract}
Developing micro- and nanomaterials for environmental pollution remediation is hot topic presently. Among the plethora of strategies, designing supported nanocatalysts for the degradation of pollutants witnessed constant renewal. In this context, we are addressing one of the UN Sustainable Development Goals by valorizing agrowaste as a source of biochar which serves as support for bimetallic nanocatalysts. Herein, Olive pit powder particles were impregnated with copper and nickel nitrates and pyrolyzed at $400{ }^{\circ} \mathrm{C}$. The resulting material consists of bimetallic $\mathrm{CuNi}$-decorated biochar. $\mathrm{CuNi}$ nanocatalysts were found to be as small as $10 \mathrm{~nm}$ and very well dispersed over biochar with zero valent copper and nickel and formation of copper-nickel solid solutions. The biochar@ $\mathrm{CuNi}$ exhibited typical soft ferromagnet hysteresis loops with zero remanence and zero coercivity. The biochar@CuNi was found to be efficient catalyst of the reduction of methyl orange (MO) dye, taken as model pollutant.

To sum up, the one pot method devised in this work provided unique $\mathrm{CuNi}$-decorated biochar and opens new horizons for the emerging topic of biochar-supported nanocatalysts.
\end{abstract}

Keywords: Olive pit; biochar; CuNi bimetallic nanocatalyst; methyl orange 


\section{Introduction}

Recently, we witnessed sky rocketing number of research papers on biochar. Search on Web of Science with keyword "biochar" returned the following results: 2 papers in 2001, 221 in 2011, 1408 in 2016, 4225 in 2020 and 1289 in 2021 (accessed 4 April 2021) which means that over 5000 papers should be published by the end of 2021 on the topic. This testifies for the vitality of this branch of materials sciences. The rationale for the rush in research on biochar lies in the availability of biomass worldwide in different forms such as agrowastes, i.e. peels [1], leaves [2], palm fronds [3] and seeds [4]. The production of biochar depends on various parameters, the initial composition of the biomass [5] and the pyrolysis parameters [6]. Biochar production falls within the energy sector as it yields bio-oil and biogas on top of the biochar itself. Biochar is employed in agriculture for soil remediation and as adsorbent in environmental applications, e.g. removal of organic and inorganic pollutants . In the domain of materials science, biochar was found to be a unique source for making graphene [7] but raised much hope as support of nanocatalysts [8]. In this latter case, biochar is usually produced then post treated for the immobilization of metal ions followed by in situ reduction therefore leading to immobilized nanocatalysts. For other purposes, metal ions are loaded on the biochar (or other carbon allotrope) and calcined to provide immobilized metal oxide nanocatalysts [9].

In order to reduce the steps to biochar-immobilized nanocatalysts, we reasoned that the initial biomass could be loaded with metal ions and the modified biomass powder could be pyrolyzed in view of obtaining, in one step, nanocatalysts-coated biochar. This approach is attractive and has been seldom reported; it concerned the design of rice husk biocharimmobilized copper metallic nanocatalysts for the catalytic cracking of biomass primary tar [10]. Olive stones are also widely agrowastes and could serve as biofillers for polymer composites [11, 12]. In 2017, some of us employed olive stone powders as biosourced cellulosic support of silver and gold nanoparticles and the final olive pit-supported nanocatalysts served for the degradation of para-nitrophenol [13]. Herein, we take advantage of this widely available agrowaste from Tunisia (a country in the top five producers of olive oil by volume [14]) to make nanocatalyst-decorated biochar. Instead of the noble metals silver and gold, we rather targeted copper-nickel bimetallic nanocatalysts. These supported bimetallic nanoparticles served as electrocatalysts for the reduction of nitrates in wastewater [15] and as heterogeneous catalysts for the reduction of dyes [16]. 
Herein, we bridge the gap between the use of olive pit powder particles as support of noble nanocatalyst and the unique, single step making of biochar-immobilized low-cost bimetallic copper-nickel nanocatalyst. As it stands, this work fulfils three requirements: $(i)$ valorization of agrowaste as biochar, (ii) biochar-supported nanocatalysts for dye degradation application, and (iii) tackling one of the United Nations' Sustainable Development Goals pertaining to environmental remediation and production of clean water.

\section{Experimental}

\subsection{Materials}

Olive pit solid waste is an industrial by-product of olive oil extraction process from Tunisia. The remaining olive oil was further treated with hexane and the remaining solid olive waste dried to evaporate water. The resulting residue was cryo-ground to fine particles (in the 60 $400 \mu \mathrm{m}$ range) [11]. The OPs employed in this work contain fat and wax: 10; pectine: 6.6; lignin: 16; hemicellulose: 43; ash: 2; and cellulose: 22.4 wt. $\%$. $\mathrm{Cu}\left(\mathrm{NO}_{3}\right)_{2}, 3 \mathrm{H}_{2} \mathrm{O}$ and $\mathrm{Ni}\left(\mathrm{NO}_{3}\right)_{2}, 6 \mathrm{H}_{2} \mathrm{O}$ were purchased from Aldrich and used as received.

\subsection{Preparation of metallic nanoparticle-coated biochar}

Weighed olive pit particles were added to metallic salt solutions under stirring. The mixtures were stirred at RT for 30 minutes and then poured over a glass lens and left to dry overnight. Due to the very hydrophobic nature of olive pit particles [13], the metal salt solutions were prepared using ethanol as solvent.

The olive pit and metal ion salt mixtures were weighed again, and when steady state mass was noticed, subjected to pyrolysis in tube furnace (Thermolyne, model 21100) under nitrogen/hydrogen mixture $(95 / 5 \%)$. Table 1 reports the experimental conditions. 
Table 1. Biochar loads and weights with copper and nickel: from the olive pit (OP) to the CuNi-decorated biochar, including pyrolysis conditions.

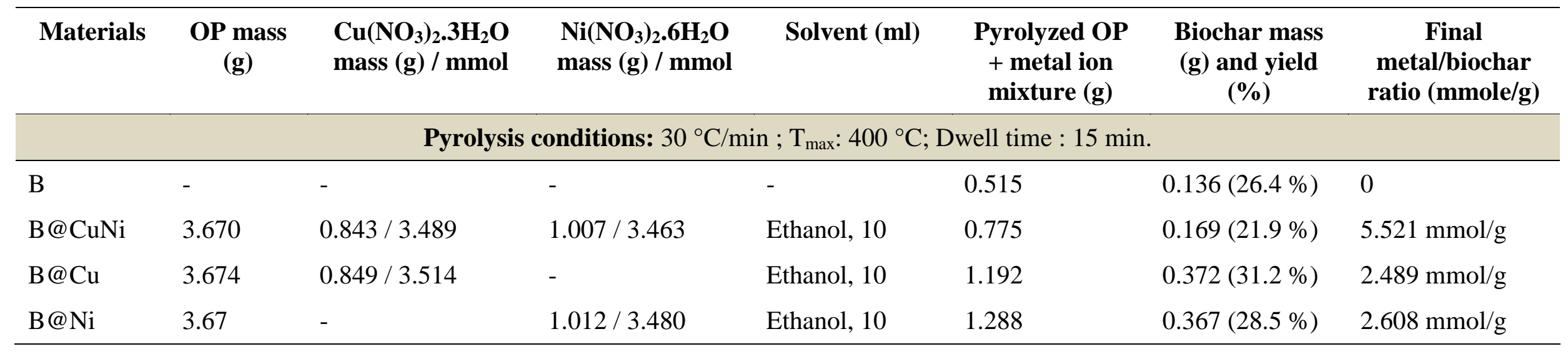




\section{Materials characterization}

SEM images and EDX spectra were acquired using a Zeiss Merlin Field Emission Scanning Electron Microscope operating at $5 \mathrm{kV}$ (Oberkochen, Germany) coupled with a SDD X-Max from Oxford Instruments. In order to avoid static charge on surface, all samples were coated with a 3,0 nm thin layer of palladium using a Cressington 208HR sputter-coater coupled with a Cressington MTM-20 thickness controller.

TGA measurements were performed with a Setaram apparatus (Setsys Evolution model). The ramp was from $\mathrm{RT}$ to $800{ }^{\circ} \mathrm{C}$ at $10^{\circ} \mathrm{C} / \mathrm{min}$ heat rate.

The XRD pattern of all the prepared samples were recorded using a X'Pert-Pro Panalytical diffractometer equipped with a Cobalt X-ray source $(\lambda=1.7889 \AA)$ and operating in the reflexion Bragg-Brentano geometry.

Magnetic measurements were conducted at room temperature on a Vibrating Sample Magnetometer (VSM) from QUANTUM DESIGN.

Biochar sample was characterized using Horiba Labram HR Evolution machine fitted with a $\mathrm{He}-\mathrm{Ne}$ laser beam the wavelength of which was set at $514 \mathrm{~nm}$.

\section{Catalysis assays}

Catalytic reduction of methyl orange was conducted in the following conditions. In a $1 \mathrm{~cm}$ length quartz cuvette, $4 \mathrm{ml}$ of $20 \mathrm{ppm}$ methylene blue solution was incubated with $1 \mathrm{mg}$ B@CuNi. The mixture was sonicated in water bath for $2 \mathrm{~min}$, then $30 \mathrm{mg} \mathrm{NaBH} 4$ were added to proceed with catalysed discoloration reaction. UV-vis spectra were recorded using Varian Cary 50 Bio apparatus.

\section{Results \& Discussion}

\subsection{General strategy of the work}

Several methods of making biochar from various sources have recently been summarized [6].The route for biochar is depicted in Figure 1a (upper panel): olive pit powder is the source of biomass, pyrolysis is the type of treatment and biochar stands for the main product. Slow and mild pyrolysis conditions were adopted: $30{ }^{\circ} \mathrm{C} / \mathrm{min}$ heating under nitrogen/ $\mathrm{H}_{2}(5 \%)$ 
atmosphere with a dwell time of 15 min at $400{ }^{\circ} \mathrm{C}$ prior to cooling at RT. $400{ }^{\circ} \mathrm{C}$ ensures a maximal biochar yield whilst $\mathrm{H}_{2}$ was employed in order to ensure metal ion reduction to metallic state. Figure 1, lower panel, displays the digital photographs of olive pit powder (Figure 1b), the same powder impregnated with copper and nickel nitrates mixture (Figure 1c), olive pit biochar (Figure 1d) and biochar-supported CuNi nanoparticles (Figure 1e). 


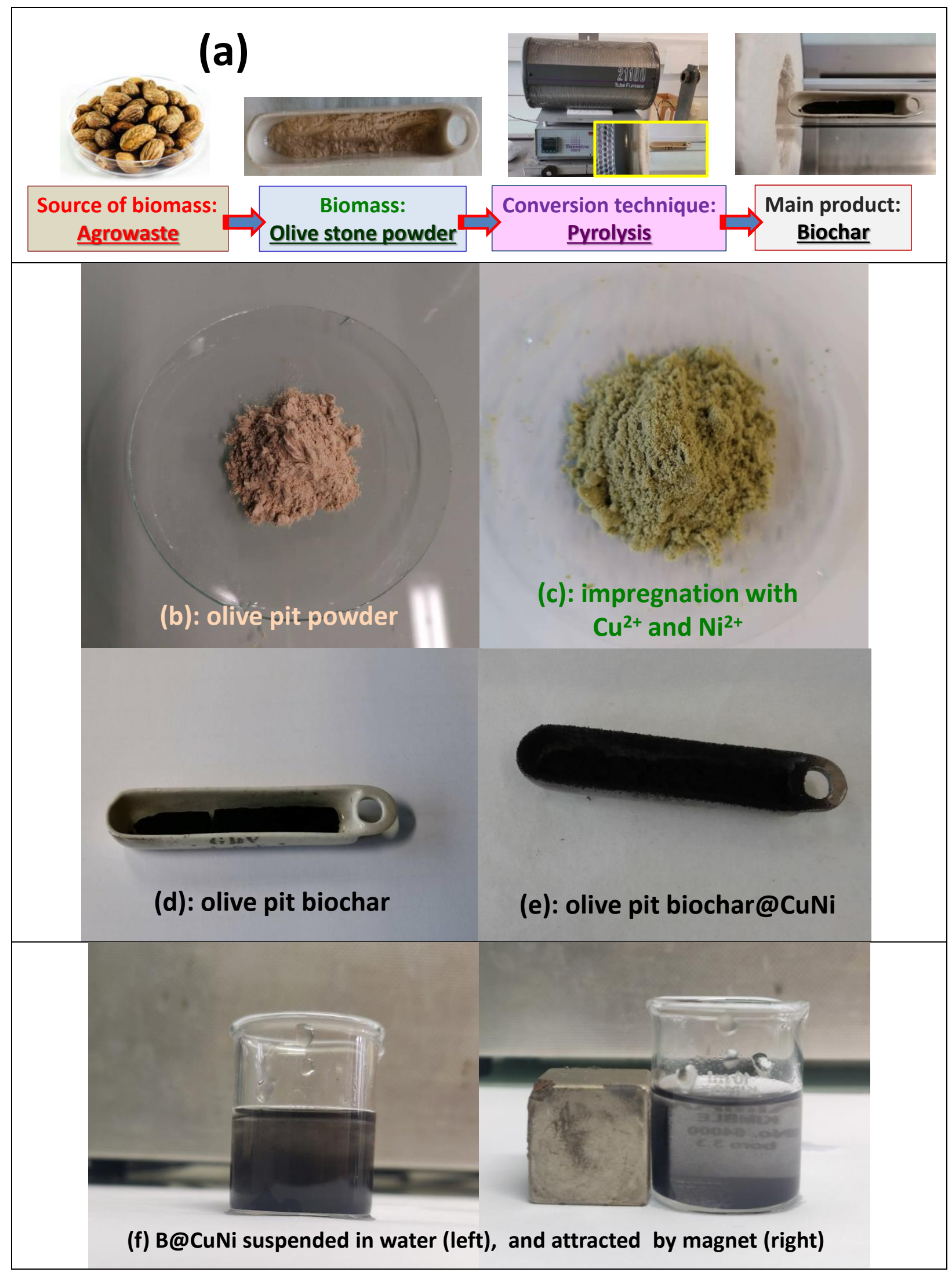

Figure 1. (a) Upper panel showing route for making biochar from olive pit biochar. Middle panel shows digital photographs of (b) olive pit powder, (c) olive pit powder after impregnation of copper and nickel nitrates, (d) the olive pit biochar, and (e) CuNi-decorated biochar obtained by direct pyrolysis of powder shown in (c). Lower panel: (f) digital pictures showing suspension of B @ CuNi before (left) and after attraction by a magnet (right). 
It is interesting to note that due to nickel, the copper-nickel alloy-decorated biochar (B@CuNi) is magnetic and could be attracted by a magnet (Figure 1f, and video as Electronic Supplementary Material ESM1).

\subsection{Surface morphology and elemental analysis}

Figure 2 displays SEM images of pristine and decorated biochar samples. Figure 2a depicts the surface morphology of $\mathrm{CuNi}$-decorated biochar. It shows a porously extending surface area for the nanocatalyst-decorated biochar. CuNi nanoparticles spread along the top of the carbonaceous sample. Upon magnifying the SEM image to provide more comprehensive information, a clearer distribution for the metallic nanoparticles can be observed as shown in Figure $2 \mathrm{~b}$. It is advantageous to notice $\mathrm{CuNi}$ nanoparticles covering the inner surface of the pores of biochar in the form of light spots onto the inner denser surface; as surrounded by a circle and ellipse in Figure 2b. CuNi nanoparticles appear as distinct spheres avoiding any aggregates that may arise in such cases as illustrated in the inset of Figure 2c. They provide spherical, undeformed structure for the provided nanoparticles. Upon comparing B@CuNi surface to that of the pristine biochar, less number of pores can be visualized on the surface as displayed in Figure 2d. These pores manifest evidently at higher magnification as illustrated in Figure 2e in a smaller proportion when compared to the CuNi-biochar sample. The inset in Figure $2 \mathrm{f}$ is mentioned here as a reference sample in the absence of $\mathrm{CuNi}$ supporting catalyst. The bare biochar micrograph denotes a plain surface without much informative morphology and its porous structure cannot be recognized easily. 


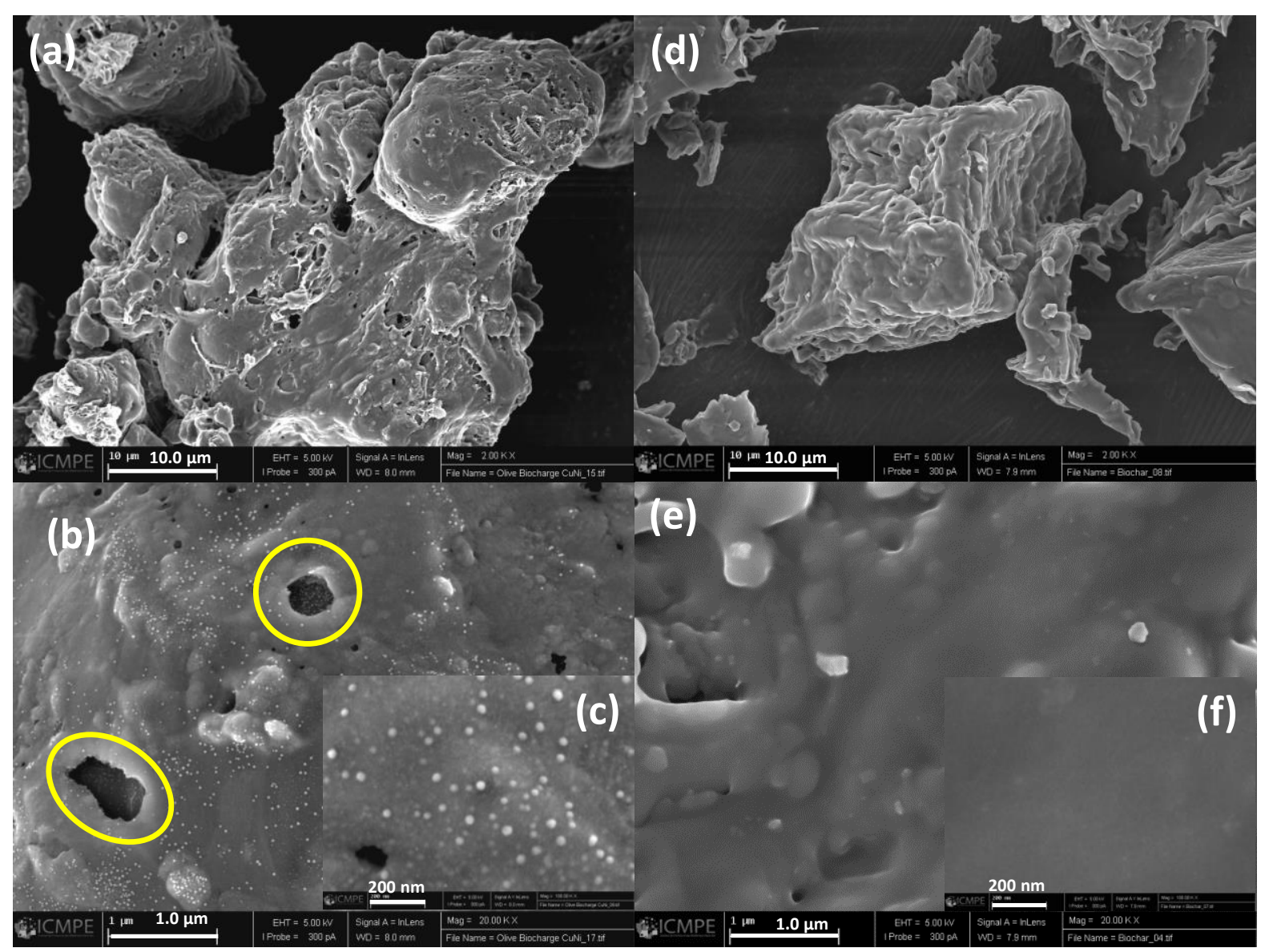

Figure 2. SEM images of biochar/CuNi hybrid (a-c) and the reference pristine biochar (d-f) without any supported nanocatalysts, at indicated magnifications. Some pores (in b) enriched with CuNi nanoparticles are surrounded by a circle and an ellipse.

EDX was used to determine the surface composition of $\mathrm{B} @ \mathrm{CuNi}$ over several spots. For comparison, we have also recorded EDX spectra for OP impregnated with metal salts, and the bare OPs. Figure 3a-c display EDX spectra of Ops, OP@metal salts and B@CuNi. Elemental composition for B@CuNi is displayed in Figure 3d, whereas $(\mathrm{Cu}+\mathrm{Ni}) /(\mathrm{C}+\mathrm{O})$ and $\mathrm{Cu} / \mathrm{Ni}$ atomic ratios are compared in Figure 3e for and OP@metal salts and B@CuNi. Figures 3a-c conclusively show the absence of any copper and nickel ions from the surface of Ops, whereas the characteristic $\mathrm{Cu}$ and $\mathrm{Ni}$ peaks are noted on the impregnated $\mathrm{OP}$ particles with peak intensity increasing in the order $\mathrm{Cu} \sim \mathrm{Ni}<\mathrm{O}<\mathrm{C}$. Increase in the $\mathrm{O} / \mathrm{C}$ peak intensity ratio is due to the hydrated metal nitrates. Upon pyrolysis, the metal nitrate-impregnated OPs are transformed into metallic alloy-decorated biochar $\mathrm{B} @ \mathrm{CuNi}$ with relative peak intensity increasing in the order $\mathrm{O}<\mathrm{Cu} \sim \mathrm{Ni}<\mathrm{C}$. Quantitatively, Figure 3d displays $\mathrm{C}, \mathrm{O}, \mathrm{Cu}$ and $\mathrm{Ni}$ atomic\% at 15 spots from $\mathrm{B} @ \mathrm{CuNi}$ powder sample; composition is fairly the same from one spot to another which indicates even decoration of the biochar with metallic nanoparticles. 
This is due to homogenous impregnation of the OPs with metal nitrates. $(\mathrm{Cu}+\mathrm{Ni}) /(\mathrm{C}+\mathrm{O})$ atomic ratio (Figure 3d) increases on going from $\mathrm{OP} @$ metal salts to $\mathrm{B} @ \mathrm{CuNi}$ due to the pyrolysis of the olive stones and subsequent release of water and nitrates. Figure $3 \mathrm{e}$ shows that $\mathrm{Cu} / \mathrm{Ni}$ atomic ratio remains the same within standard deviation for OP@metal salts

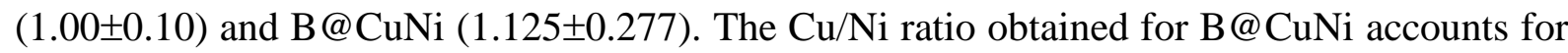
the equimolar impregnation of olive pits by metal ions.

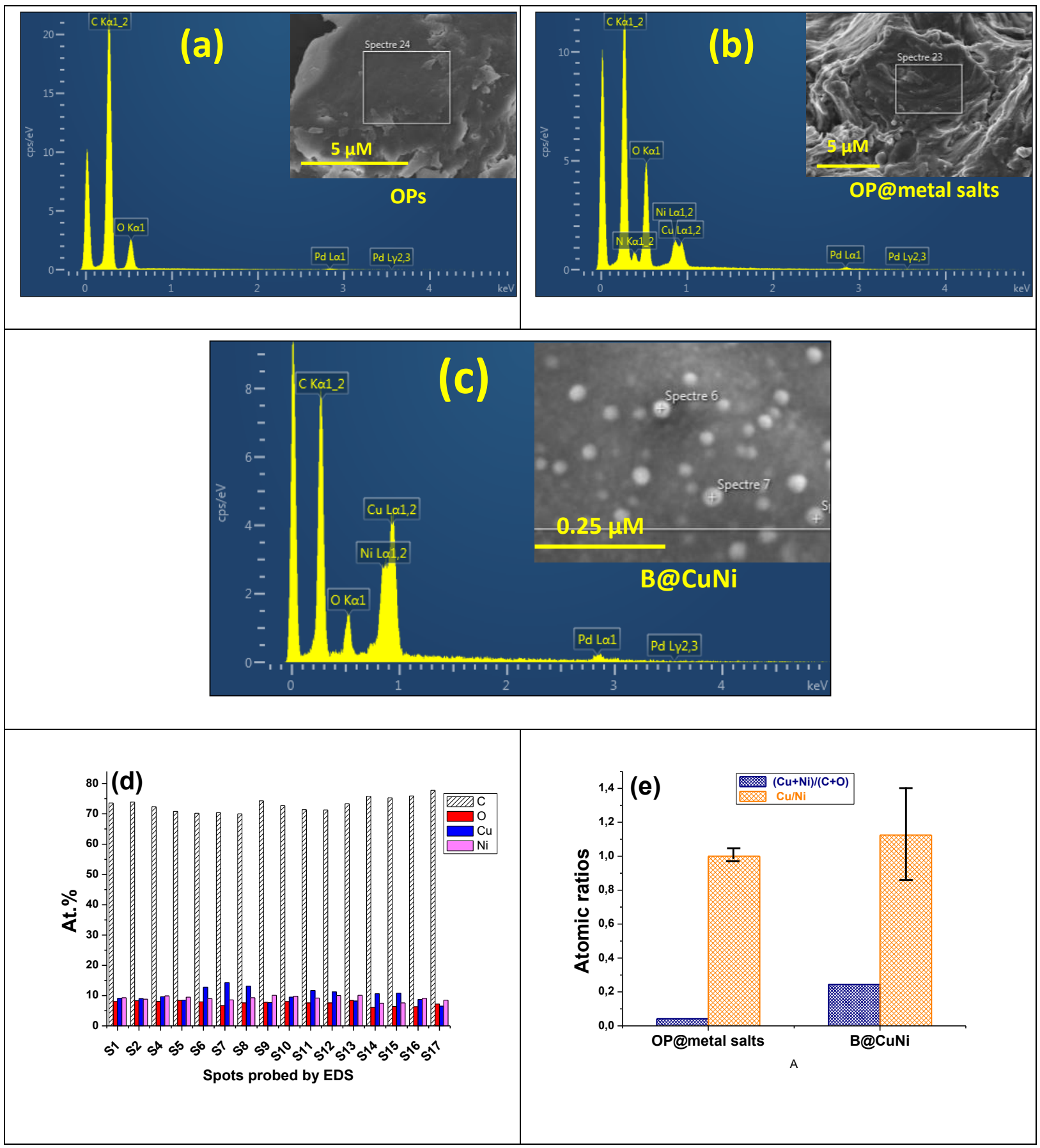

Figure 3. EDX spectra of (a) olive pit powder particles, OPs; (b) Ops impregnated with metal salts (copper and nickel nitrates), and (c) B@CuNi. SEM images show spots probed for the acquisition of EDX spectra. EDX quantification: (d) elemental analysis of $\mathrm{C}, \mathrm{O}, \mathrm{Cu}$ and Ni detected at 15 spots of B@CuNi surface; and (e) $(\mathrm{Cu}+\mathrm{Ni}) /(\mathrm{C}+\mathrm{O})$ and $\mathrm{Cu} / \mathrm{Ni}$ atomic ratios averaged over 15 spots. 


\subsection{Phase analysis}

The prepared catalysts were analysed using both XRD and Raman to identify the nature of the metal and carbon contents, respectively. Figure 4 displays XRD patterns of B@CuNi and of the reference materials Biochar and B@Ni. Those of B@Ni and B@Cu (not shown) correspond very well to the structures of nickel and copper face centered cubic metals (ICDD n98-004-1508 and 98-005-2256), respectively. The XRD pattern of B@CuNi exhibits the same type of diffraction lines but at $2 \theta$ positions located between those of pure $\mathrm{Cu}$ an pure $\mathrm{Ni}$ phases, suggesting that the biochar serves as support of a $\mathrm{Ni}_{1}-\mathrm{XCu}_{\mathrm{x}}$ metallic solid solution. This is strong supporting evidence that copper and nickel were alloyed during the one-step pyrolysis process. Moreover, it is noteworthy that all peaks are broadened which is in line with the production of small crystals that were imaged by SEM (see Figure 2b,c).

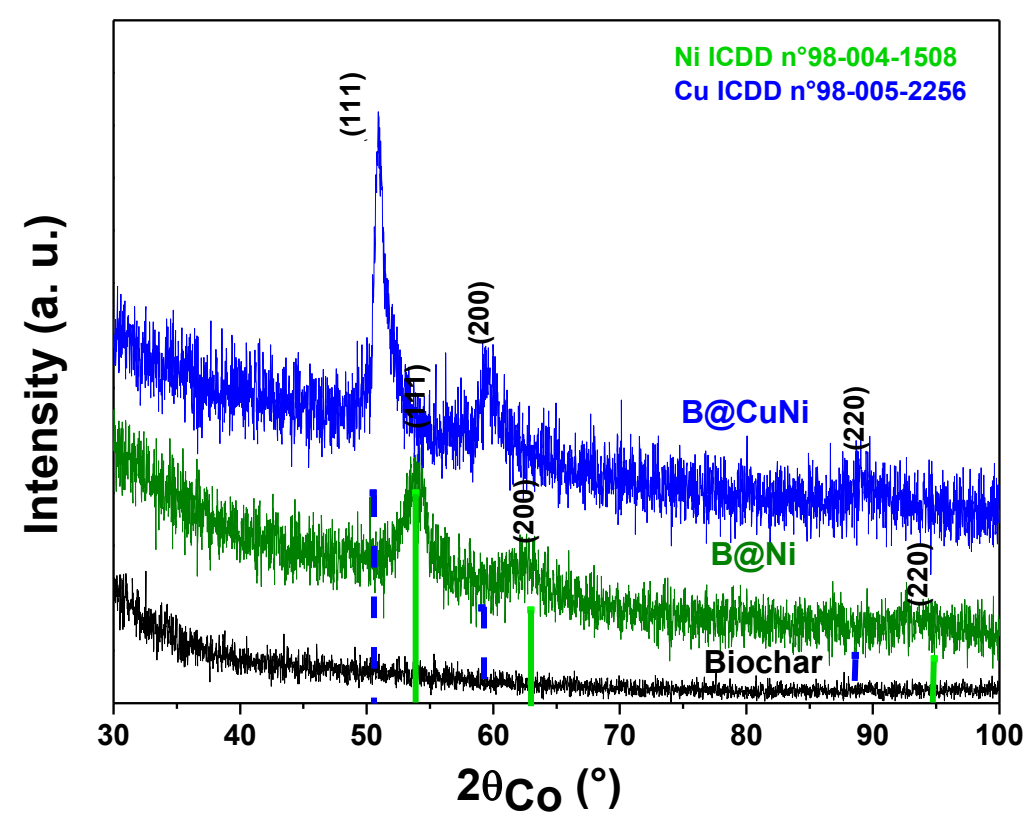

Figure 4. XRD-patterns of $\mathrm{B} @ \mathrm{Ni}$ and $\mathrm{B} @ \mathrm{CuNi}$ samples compared to that of free biochar. The tabulated peak positions of bulk $\mathrm{Ni}$ (ICDD $\mathrm{n}^{\circ}$ 98-004-1508) and $\mathrm{Cu}$ phases (98-005-2256) are given for information.

The formation of metallic $\mathrm{Ni}$ and $\mathrm{CuNi}$ particles was also confirmed by the measurement of the variation of the sample magnetization $\mathrm{M}$ as a function of the magnetic field $\mathrm{H}$ at room temperature. Indeed, by cycling the magnetic field $\mathrm{H}$ between -20.0 and $+20.0 \mathrm{kOe}$, typical soft ferromagnet hysteresis loops with zero remanence and zero coercivity [17], were recorded on both $\mathrm{B} @ \mathrm{CuNi}$ and $\mathrm{B} @ \mathrm{Ni}$ samples (Figure 5). This feature must be underlined since it accounts for the capability of the designed catalysts to be reversibly attracted by an external magnetic field making their recovering and reuse easy to achieve. Interestingly, as the copper alloyed nickel particles are usually less magnetic than the nickel pure one, one may notice on the recorded $\mathrm{M}(\mathrm{H})$ plots that the saturation magnetization of $\mathrm{B} @ \mathrm{Ni}$ is much more quickly reached than that of $\mathrm{B} @ \mathrm{CuNi}$. 


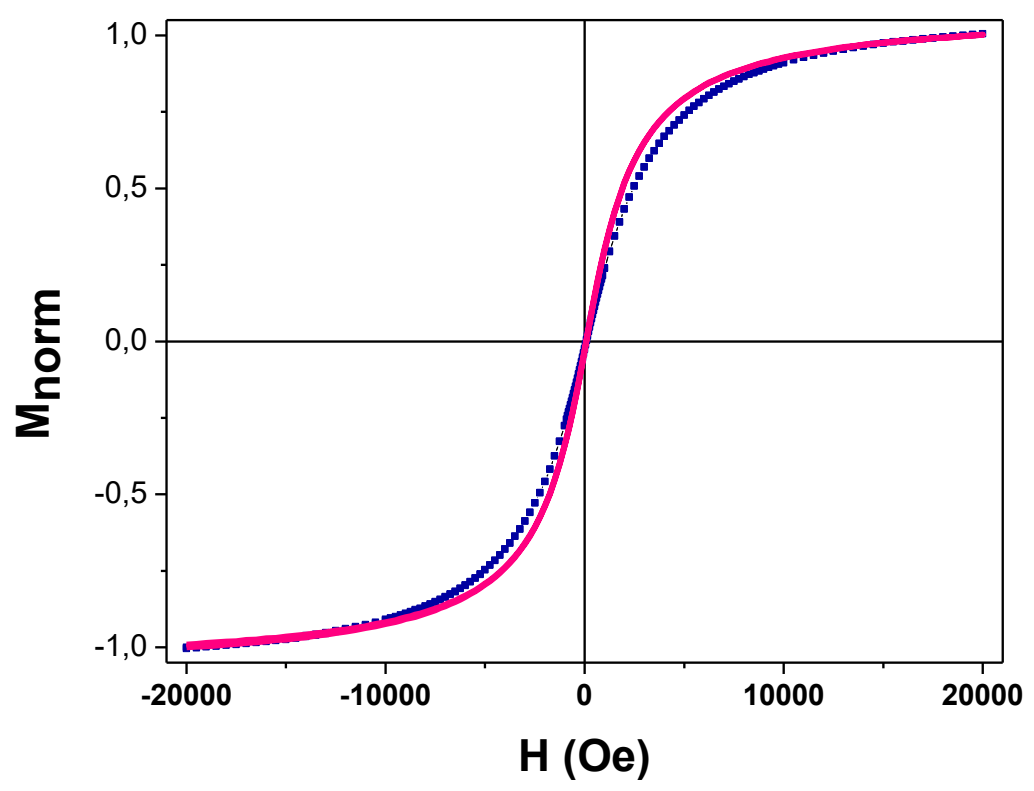

Figure 5. Variation of the normalized magnetization as a function of the magnetic field at room temperature on B@ $\mathrm{Ni}(-)$ and $\mathrm{B} @ \mathrm{CuNi}(\boldsymbol{\square})$.

To complete these structural characterizations, Raman spectroscopy was carried out on the prepared sample with a special emphasis on their carbon signature. The spectrum recorded in the $1000-2000 \mathrm{~cm}^{-1}$ energy region, on the metal free Biochar is shown in Figure 6 to illustrate this purpose. It is fitted with five components assigned to $S\left(1262 \mathrm{~cm}^{-1} ; \mathrm{sp}^{2}-\mathrm{sp}^{3}\right), \mathrm{D}\left(1380 \mathrm{~cm}^{-}\right.$ ${ }^{1}$; , V (1506 $\mathrm{cm}^{-1} ; \mathrm{sp}^{2}-\mathrm{sp}^{3}$, amorphous carbon structures $), \mathrm{G}\left(1596 \mathrm{~cm}^{-1}\right.$; graphite, $\left.\mathrm{sp}^{2}\right)$ and $\mathrm{G}_{\mathrm{L}}$ $\left(1696 \mathrm{~cm}^{-1} ; \mathrm{sp}^{2}, \mathrm{C}=\mathrm{O}\right)[18,19]$. Particularly, the $\mathrm{D}$ and $\mathrm{G}$ bands are assigned to disordered and ideal graphitic lattice, respectively. The D/G peak height and ratios area ratios equal 0.66 and 0.87 , respectively. The spectrum indicates therefore the presence of $\mathrm{C} \mathrm{sp}{ }^{2}$ atoms, but without any excess of amorphous $\mathrm{sp}^{3}$ hydrocarbons. It is to note that the $\mathrm{D} / \mathrm{G}$ ratio, referring to the degree of defects in the carbon material, is much lower than those reported for hydrothermally synthesized graphene quantum dots [20]. It is also lower than the values reported for pruning wastes of apple trees post-treated at $400{ }^{\circ} \mathrm{C}(\mathrm{D} / \mathrm{G}$ height ratio $\sim 0.72)$ [21], ferrate-activated porous biochar prepared at $900{ }^{\circ} \mathrm{C}(\mathrm{D} / \mathrm{G}$ height ratio= 1.07$)[22]$, and $(\mathrm{D} / \mathrm{G}$ height ratio $=1.14)$ [23]. This is important when considering low pyrolysis temperature and high yield we have obtained, as well as relatively higher graphitization. 


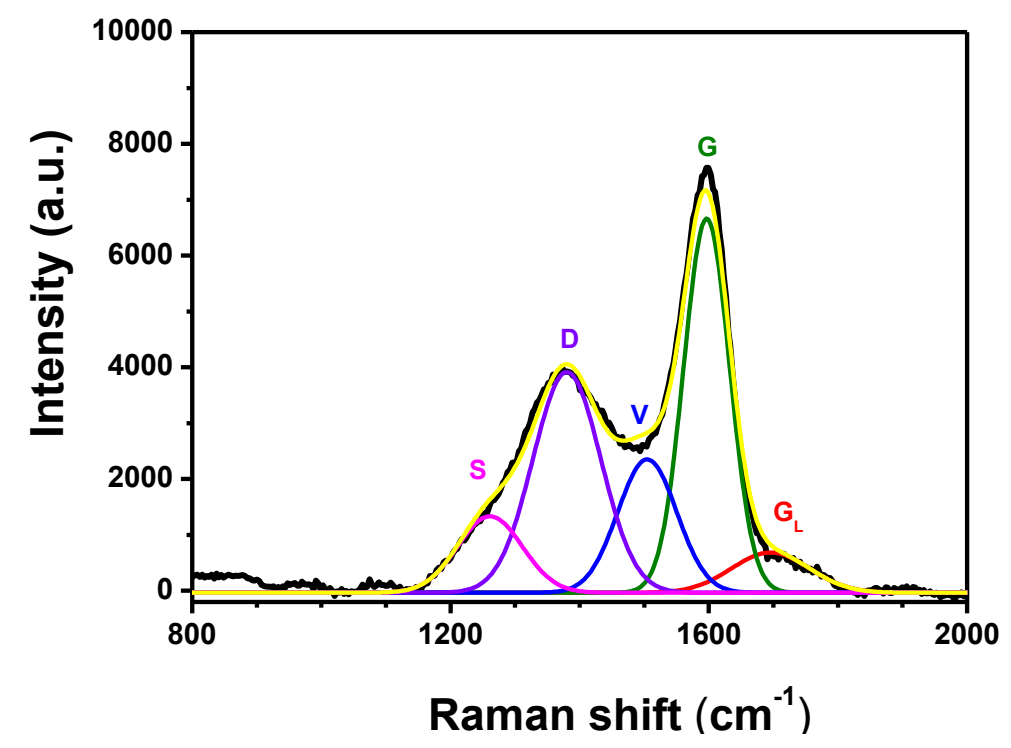

Figure 6. Peak-fitted Raman spectrum of olive pit biochar.

\subsection{Thermal stability}

Thermogravimetric analysis (TGA) was utilized as a substantial technique to monitor the thermal stability of biochar and match it with $\mathrm{CuNi}$-decorated biochar. Both samples show a comparable behaviour of thermal stability till reaching $240{ }^{\circ} \mathrm{C}$ for steady samples withstanding temperature as shown in Figure 4. Thermogravimetric analysis was carried under air. A slight increase of the weight of the pristine biochar sample is observed at $280{ }^{\circ} \mathrm{C}$. This can be correlated to the interaction of this sample with the atmospheric oxygen. It might have influenced the biochar specimen and led to a slight increase in its weight\%. At higher temperatures, biochar started to decompose at $330-340{ }^{\circ} \mathrm{C}$ till reaching $500{ }^{\circ} \mathrm{C}$.

For $\mathrm{B} @ \mathrm{CuNi}$, a plateau region was reached and corresponds to $42 \%$ residual weight of the original sample. The sample retained its thermal stability up to $270-280{ }^{\circ} \mathrm{C}$ then a decomposition step occurred at elevated temperatures to reach steady state at $455{ }^{\circ} \mathrm{C}$ with a residual weight of $45 \%$ of the original weight, higher than that of biochar due to loaded metallic nanoparticles [16]. 


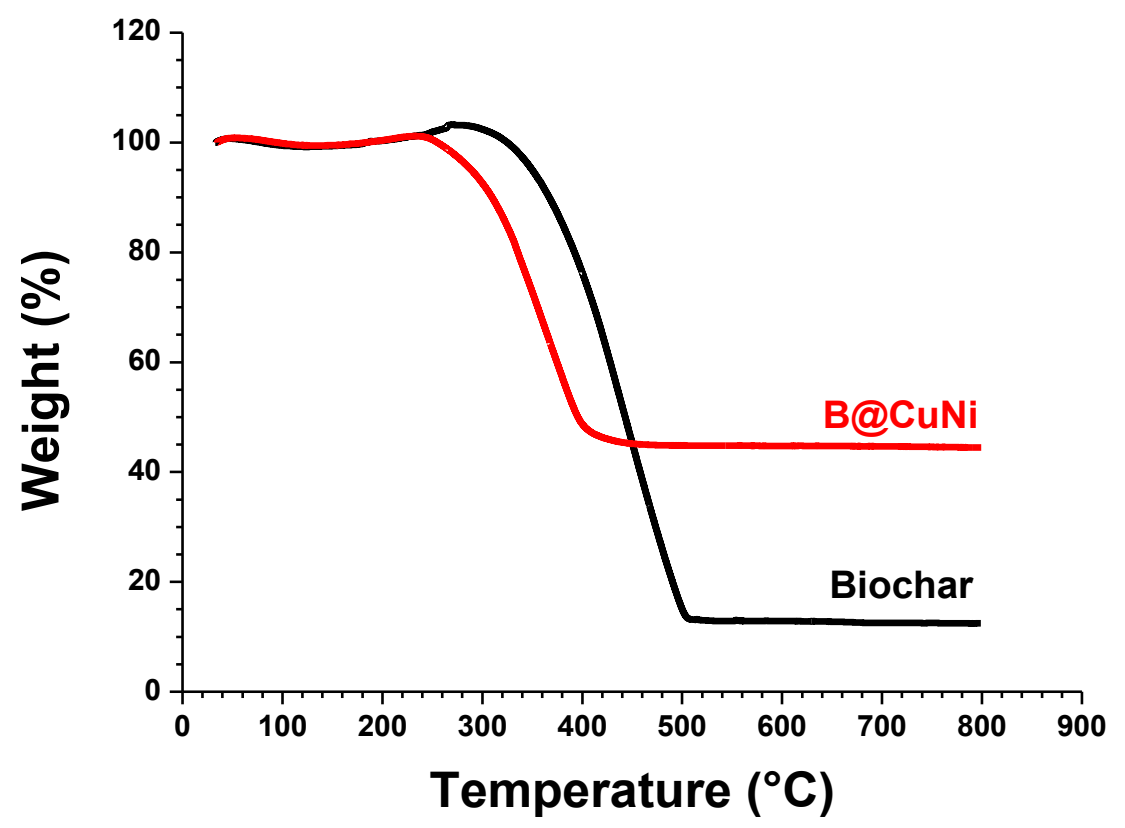

Figure 4. TGA plots recorded under air for biochar and B@CuNi.

\subsection{Potential application: heterogeneous catalyzed degradation of methyl orange dye}

Dye removal can be achieved through various routes comprising adsorption [24], filtration [25] or catalyzed degradation [26, 27]. Herein, we focus on the catalyzed degradation of MO in aqueous solution in order to assess the catalytic activity of the actual CuNi-decorated biochar. This process was carried out in the presence of $\mathrm{NaBH}_{4}$ as reducing agent. Upon starting at time $=0 \mathrm{sec}$, the investigated samples did not show any significant changes in color of tested vials as illustrated in the upper row in Fig. 7a. After 30 min, the color of the solution loaded with decorated biochar turned to be colorless referring to decomposed MO (Fig. 7b). UV-vis spectra of MO with different reactants in the presence and absence of the decorated biochar are illustrated in Fig. 7c. Starting with the spectrum showing peaks with the highest intensity among the explored samples, MO displays a typical intense peak at $464 \mathrm{~nm}$ accompanied with a lower one at $270 \mathrm{~nm}$. The first peak can be correlated to the conjugation of electron donor $\mathrm{N}=\mathrm{N}$ bond. Meanwhile, the latter peak is featured to aromatic $\pi-\pi^{*}$ transition [28]. After diluting MO with deionized water, the same behaviour for absorbance bands arose, however with lower peak intensities. This may be referred to the dilution effect resulting from introducing water to the previously tested MO aqueous solution. The dilution step was implemented to confirm the presence of $\mathrm{MO}$ in the solution even at lower concentration with weaker absorption bands. Upon investigating the samples of MO with 
either $\mathrm{CuNi}$-decorated biochar or $\mathrm{NaBH}_{4}$ separately, the absorption peaks showed neither significant changes, nor shifts. Upon testing $\mathrm{MO}$ with $\mathrm{B} @ \mathrm{CuNi}$ and $\mathrm{NaBH}_{4}$, the solution became transparent; this testifies for the degradation process of MO. The previously mentioned peaks vanished and new bands appeared at 245 and $248 \mathrm{~nm}$. Accordingly, a cleavage of $-\mathrm{N}=\mathrm{N}$ - bond took place to generate $-\mathrm{NH}_{2}$ group. Hence, the newly formed species, namely sulfanilic acid and p-amino dimethylaniline, respectively [29, 30]. It is to note that no centrifugation was required to split the biochar and the supernatant; the latter was sedimented easily using a magnet, which facilitated sampling the supernatant for UV-vis analysis.

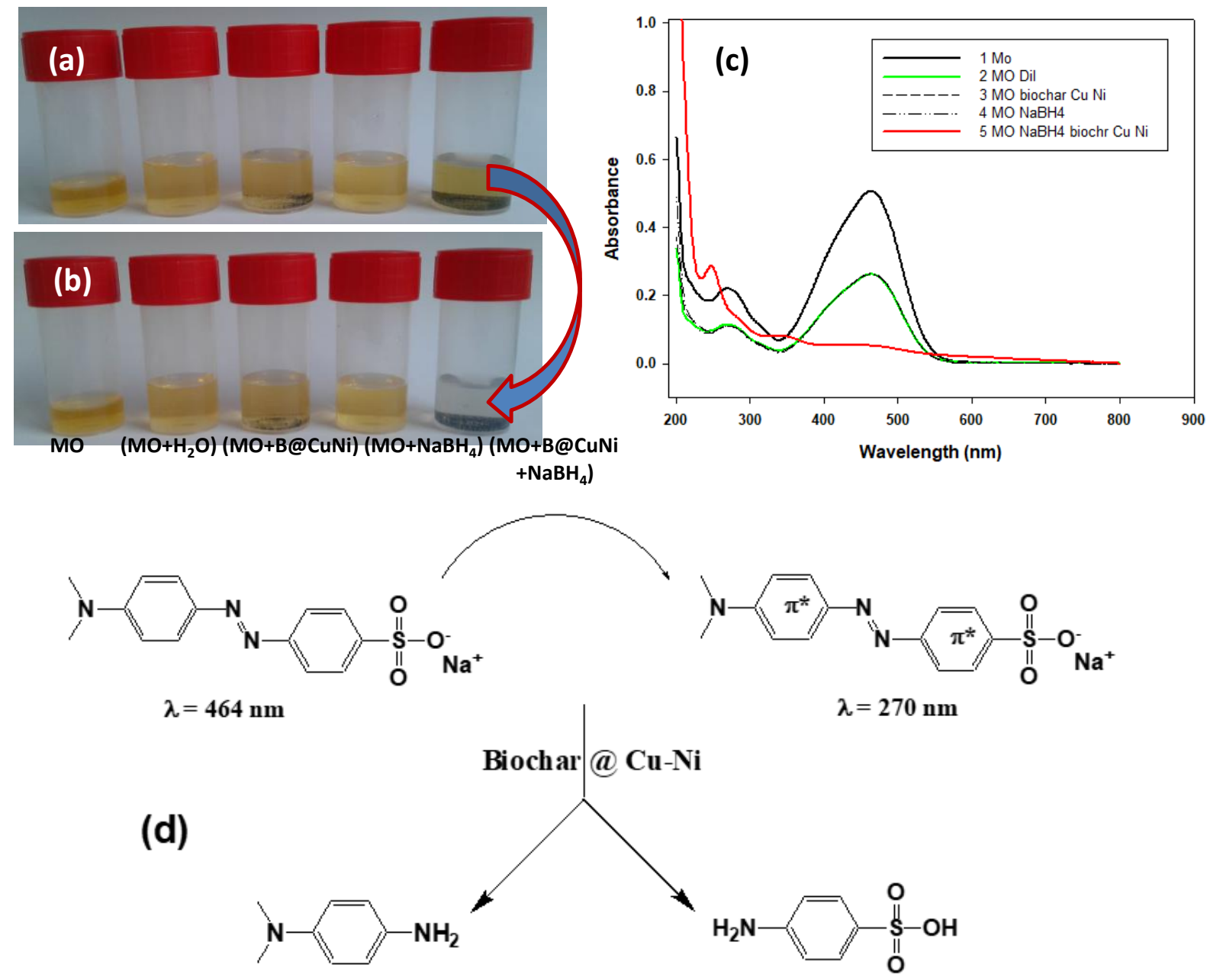

Figure 7. (a,b) Digital photographs and (b) UV-vis spectra for MO vials before and after catalytic reaction. (d) A proposed scheme for MO before and after the catalytic degradation with decorated CuNi-biochar. 


\section{Conclusion}

We have composed a boosted biochar derived from an agrowaste; olive pit (OP) powder. Metallic salts of hydrated nickel and/or copper nitrate(s) as precursors were mixed with OP followed by pyrolysis, in one step at $400{ }^{\circ} \mathrm{C}$ to generate biochar@CuNi (B@CuNi). The resulting biochar was characterized by (SEM) to show a homogenous surface with well distributed CuNi nanoparticles onto the surface of the biochar. EDX and XRD asserted the formation of $\mathrm{CuNi}$ nanoparticles as metallic alloy decorating the biochar. Magnetization experiments accounted for the presence of nickel and demonstrated that nanocatalystdecorated biochars behave as ferromagnets. B@CuNi showed a high catalytic performance in decomposing methyl orange (MO) dye. The significance of this work is to produce an enhanced biochar decorated with bimetallic nanocatalyst $(\mathrm{CuNi})$ in a facile process. This strategy of making nanocatlyst-doped biochar opens new avenues for water treatment and therefore, attempting to elegantly contribute in environmental remediation.

\section{Acknowledgements}

AMK and MMC would like to thank both the French and Egyptian Governments for funding AMK's contribution through a fellowship granted by the French Embassy in Egypt (Institut Francais d'Egypte) and Science and Technology Developing Fund (STDF)-Egypt, Project number (42248). Mrs Stépahnie Lau-Truong is acknowledged for Raman analysis.

Conflict of interest: the authors declare no conflict of interest

Electronic Supplementary Material ESM1: https://vimeo.com/558456241

\section{References}

1. Adeniyi, A.G., J.O. Ighalo, and D.V. Onifade, Biochar from the thermochemical conversion of orange (Citrus sinensis) peel and Albedo: product quality and potential applications. Chemistry Africa, 2020. 3(2): p. 439-448.

2. Azeem, M., et al., Tea leaves biochar as a carrier of Bacillus cereus improves the soil function and crop productivity. Applied Soil Ecology, 2021. 157: p. 103732.

3. Zubair, M., et al., Adsorption behavior and mechanism of methylene blue, crystal violet, Eriochrome black T, and methyl orange dyes onto biochar-derived date palm 
fronds waste produced at different pyrolysis conditions. Water, Air, \& Soil Pollution, 2020. 231: p. 1-19.

4. Bagheri, A., et al., Modified biochar from Moringa seed powder for the removal of diclofenac from aqueous solution. Environmental Science and Pollution Research, 2020. 27(7): p. 7318-7327.

5. Sun, X., et al., Characterization of 60 types of Chinese biomass waste and resultant biochars in terms of their candidacy for soil application. Gcb Bioenergy, 2017. 9(9): p. 1423-1435.

6. Tripathi, M., J.N. Sahu, and P. Ganesan, Effect of process parameters on production of biochar from biomass waste through pyrolysis: A review. Renewable and Sustainable Energy Reviews, 2016. 55: p. 467-481.

7. Luong, D.X., et al., Gram-scale bottom-up flash graphene synthesis. Nature, 2020. 577(7792): p. 647-651.

8. $\quad$ Lopes, R.P. and D. Astruc, Biochar as a support for nanocatalysts and other reagents: Recent advances and applications. Coordination Chemistry Reviews, 2021. 426: p. 213585.

9. Tan, G., et al., Removal of Pb (II) ions from aqueous solution by manganese oxide coated rice straw biochar A low-cost and highly effective sorbent. Journal of the Taiwan Institute of Chemical Engineers, 2018. 84: p. 85-92.

10. Guo, F., et al., One-step synthesis of biomass activated char supported copper nanoparticles for catalytic cracking of biomass primary tar. Energy, 2019. 180: p. 584-593.

11. Khemakhem, M. and M. Jaziri, Composites based on (ethylene-propylene) copolymer and olive solid waste: rheological, thermal, mechanical, and morphological behaviors. Polymer Engineering \& Science, 2016. 56(1): p. 27-35.

12. Khalil, A.M., K.F. El-Nemr, and M.L. Hassan, Acrylate-modified gamma-irradiated olive stones waste as a filler for acrylonitrile butadiene rubber/devulcanized rubber composites. Journal of Polymer Research, 2019. 26(11): p. 1-11.

13. Belbekhouche, S., et al., Aryl diazonium-modified olive waste: A low cost support for the immobilization of nanocatalysts. Colloids and Surfaces A: Physicochemical and Engineering Aspects, 2017. 529: p. 541-549.

14. Fernández-Uclés, D., et al., Economic Efficiency in the Tunisian Olive Oil Sector. Agriculture, 2020. 10(9): p. 391.

15. Mirzaei, P., Préparation de matériaux d'électrode pour l'élimination et la valorisation de polluants azotés. 2018, Paris Est.

16. Ahsan, M.A., et al., Ultrafast catalytic reduction of environmental pollutants in water via MOF-derived magnetic Ni and Cu nanoparticles encapsulated in porous carbon. Applied Surface Science, 2019. 497: p. 143608.

17. Luo, M., et al., Removal of aqueous Cr (VI) using magnetic-gelatin supported on Brassica-straw biochar. Journal of Dispersion Science and Technology, 2020: p. 1-13.

18. Shao, Y., et al., Biocarbons from microfibrillated cellulose/lignosulfonate precursors: A study of electrical conductivity development during slow pyrolysis. Carbon, 2018. 129: p. 357-366.

19. Li, X., J.-i. Hayashi, and C.-Z. Li, Volatilisation and catalytic effects of alkali and alkaline earth metallic species during the pyrolysis and gasification of Victorian brown coal. Part VII. Raman spectroscopic study on the changes in char structure during the catalytic gasification in air. Fuel, 2006. 85(10-11): p. 1509-1517.

20. Xiao, X. and B. Chen, A direct observation of the fine aromatic clusters and molecular structures of biochars. Environmental science \& technology, 2017. 51(10): p. 5473-5482. 
21. Kim, D.-G. and S.-O. Ko, Effects of thermal modification of a biochar on persulfate activation and mechanisms of catalytic degradation of a pharmaceutical. Chemical Engineering Journal, 2020. 399: p. 125377.

22. Thi Minh Tam, N., et al., Efficient removal of diclofenac from aqueous solution by potassium ferrate-activated porous graphitic biochar: ambient condition influences and adsorption mechanism. International journal of environmental research and public health, 2020. 17(1): p. 291.

23. Endler, L.W., et al., Facile method to prepare biochar-NiO nanocomposites as a promisor material for electrochemical energy storage devices. Chemical Papers, 2020. 74(5): p. 1471-1476.

24. Khalil, A.M. and S.H. Kenawy, Hybrid Membranes Based on Clay-Polymer for Removing Methylene Blue from Water. Acta Chimica Slovenica, 2020. 67(1): p. 96104.

25. Abdelhamid, A.E., A.A. El-Sayed, and A.M. Khalil, Polysulfone nanofiltration membranes enriched with functionalized graphene oxide for dye removal from wastewater. Journal of Polymer Engineering, 2020. 40(10): p. 833-841.

26. Ait-Touchente, Z., et al., Ultrasonic effect on the photocatalytic degradation of Rhodamine $6 G(R h 6 G)$ dye by cotton fabrics loaded with TiO 2. Cellulose, 2020. 27(2): p. 1085-1097.

27. Mousli, F., et al., Mixed oxide-polyaniline composite-coated woven cotton fabrics for the visible light catalyzed degradation of hazardous organic pollutants. Cellulose, 2020. 27(13): p. 7823-7846.

28. Yang, L., et al., Self-assembly montmorillonite nanosheets supported hierarchical MoS2 as enhanced catalyst toward methyl orange degradation. Materials Chemistry and Physics, 2020. 246: p. 122829.

29. Bakhsh, E.M., et al., Performance of cellulose acetate-ferric oxide nanocomposite supported metal catalysts toward the reduction of environmental pollutants. International journal of biological macromolecules, 2018. 107: p. 668-677.

30. Sha, Y., et al., Rapid degradation of azo dye methyl orange using hollow cobalt nanoparticles. Chemosphere, 2016. 144: p. 1530-1535. 